\title{
PeRSONALIDAD Y LA CONDUCCIÓN DE LA VIDA COTIDIANA
}

\author{
Ole Dreier \\ Departament of Psychology, University of Copenhagen \\ Dinamarca
}

Traducido de:

\section{PERSONALITY AND THE CONDUCT OF EVERYDAY LIFE}

\author{
Nordic Psychology \\ 2011 \\ pp.4-23 \\ Traducción realizada por: \\ Juan José Yoseff-Bernal
}

\section{RESUMEN}

El propósito principal de este escrito es presentar una teoría sobre las personas que está arraigada en la manera en la que las personas conducen sus vidas cotidianas. La aproximación y los conceptos clave en la teoría son presentados en la segunda y central parte del escrito. Esta aproximación teórica sobre la personalidad es inusual. La investigación actual sobre la personalidad reconoce que debe ser estudiada en la interacción entre persona-situación-comportamiento. En la primera parte de este escrito presento algunas de las cuestiones centrales en esos estudios. La aproximación teórica busca resolver tales cuestiones. Estas cuestiones son una de las dos mayores fuentes de inspiración para la aproximación teórica aquí propuesta. La otra fuente importante de inspiración son los estudios sobre intervenciones psicológicas. En la tercera parte del escrito presento un estudio que da luz sobre cómo la teoría puede provocar y enriquecer nuestro entendimiento de las intervenciones.

Palabras clave

conducción de vidas cotidianas, persona, personalidad, persona-situación-conducta, intervenciones psicológicas

Bitácora del Artículo:

| Recibido: 4 de Febrero de 2015 | Aceptado: 4 de Marzo de 2015 | Publicado en línea: Enero-Junio de 2016 


\title{
Autoría y Derechos de Propiedad Intelectual
}

\section{PERSONALIDAD Y LA CONDUCCIÓN DE LA VIDA COTIDIANA}

\author{
Ole Dreier \\ Departament of Psychology, University of Copenhagen \\ Dinamarca
}

Traducido de:

\section{PERSONALITY AND THE CONDUCT OF EVERYDAY LIFE}

\section{Nordic Psychology}

2011

pp.4-23

Traducción realizada por:

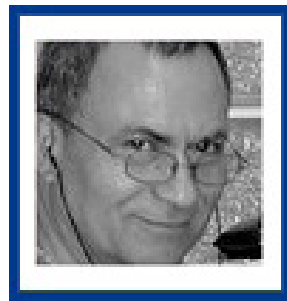

Juan José Yoseff-Bernal

UNAM - FES Iztacala

Correo: jyoseff@gmail.com

Licenciado y Maestro en Psicología por la Facultad de Estudios Superiores Iztacala, Doctor en Psicología por la Universidad de Sevilla.

Ver más...

\section{DATOS DE FILIACIÓN DEL TRADUCTOR}

Profesor titular FES Iztacala, UNAM

\section{(c) (i) (2)(2)}

Copyright: (C) 2016 Yoseff-Bernal, J. J.

Este es un artículo de acceso abierto distribuido bajo los términos de la licencia Creative Commons Reconocimiento-NoComercial -Compartirlgual 4.0 Internacional, por lo que su contenido gráfico y escrito se puede compartir, copiar y redistribuir total o parcialmente sin necesidad de permiso expreso de su autor con la única condición de que no se puede usar con fines directamente comerciales y los términos legales de cualquier trabajo derivado deben ser los mismos que se expresan en la presente declaración. La única condición es que se cite la fuente con referencia a la Revista Digital Internacional de Psicología y Ciencia Social y a su autor.

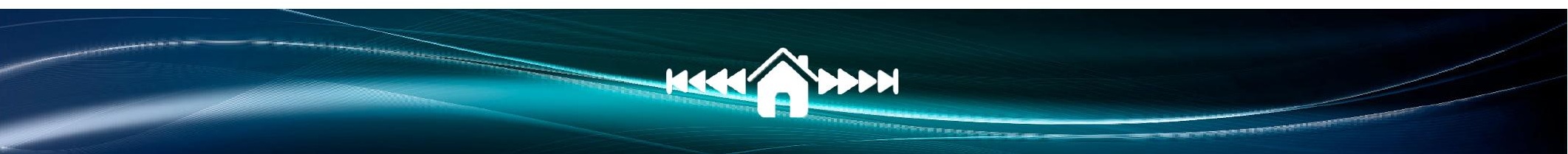




\section{TABLA DE CONTENIDO}

INTRODUCCIÓN

1. Asuntos CENTRALES NO RESUELTOS EN LA INVESTIGACIÓN SOBRE LA PÉRSONALIDAD

1.1 Persona-situación-comportamiento, 4

\subsection{PERSONA-SITUACIÓN-COMPORTAMIENTO EN LA VIDA COTIDIANA}

2. UnA PROPUESTA TEÓRICA

2.1 Orden y arreglos, 7

2.2 Participación situada y movimiento, 8

2.3 Conducción de la vida cotidiana, 9

3. IMPLICACIONES PARA ENTENDER LAS INTERVENCIONES PSICOLÓGICAS

3.1 Arreglo de intervenciones a través de contextos, 11

3.2 Búsqueda de cambios a través de contextos, 11

3.3 Intervenciones en las vidas cotidianas, 13

3.4 Intervenciones y conducción de la vida cotidiana, 13

4. ConClusión

REFERENCIAS 
INTRODUCCIÓN

\section{Asuntos centrales no resueltos en la INVESTIGACIÓN SOBRE LA PERSONALIDAD}

\subsection{Persona-situación-comportamiento}

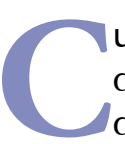

ualquier enunciado acerca de la personalidad descansa en tres clases de elementos: a) factores de la personalidad, b) la situación en la cual estos factores se estudian o se predice su manifestación, y c) el comportamiento en el cual estos factores de personalidad se expresan y lo que les afecta. En consecuencia, la consciencia se manifiesta en este tipo de comportamiento en tal situación.

Con mucha frecuencia el debate acalorado sobre la interrelación persona-situación en la psicología de la personalidad ha llevado a un acuerdo común de que debemos estudiar la personalidad en relación a las situaciones y al comportamiento. Pero no hay acuerdo en cómo medir empíricamente las relaciones entre personalidad-situación-comportamiento; ni hay acuerdo en el peso teórico dado a cada uno de los aspectos; como tampoco hay acuerdo en cómo explicar la interrelación entre esos tres aspectos.

Aquí me valdré de un análisis crítico de los asuntos clave en la investigación reciente sobre la interrelación persona-situación-comportamiento para mostrar que la conducción de una persona en la vida cotidiana juega un rol central en la personalidad.

Mischel es una figura central en el debate persona-situación (e.g., Mischel, 1968; Mischel, 2004; Mischel y Shoda, 2008). En un estudio clásico de Shoda,
Mischel y Wright (1994) se observó a 84 niños por 150 horas en un campamento de verano. Shoda y sus colegas analizaron sus comportamientos agresivos y concluyeron que cada niño se caracterizaba por un rasgo de personalidad comportamental distintivo. Un niño actuaba agresivamente cuando sus compañeros lo molestaban. Otro actuó agresivamente cuando fue prevenido por un adulto. Shoda y sus colegas sostienen que los patrones estables individuales emergieron de la historia de los niños de ser sensibilizados a características particulares de situaciones. Al investigar vínculos entre persona-situación-comportamiento debemos por lo tanto identificar cuales características de las situaciones son psicológicamente importantes. La Tabla 1 de su estudio muestra cómo Shoda y sus colegas hicieron esto (ibid., p. 676). Leyendo la tabla de izquierda a derecha podemos ver que ellos consideraron una "escena" como sostener "situaciones nominales" que fueron definidas por "la estructura de una ecología dada (la escena), más que por su impacto psicológico potencial sobre y el significado para la persona" (ibid., p. 675). También insisten en que sólo los eventos psicológicos interpersonales particulares contenidos en esas situaciones nominales eran características de las situaciones psicológicamente importantes. Por ejemplo, trabajar la madera es "una situación nominal que contiene tan diversos eventos psicológicos interpersonales como ser elogiado, frustrado, molestado, y castigado" (ibid., p. 675). Como se pueden encontrar los mismos eventos en muchas situaciones nominales, de acuerdo con su aproximación, pueden crearse catálogos de tipos generales de eventos interpersonales a través de las situaciones. De esta manera, Shoda y sus colegas construyeron un $A B C$ de eventos interpersonales que se mantuvieron inafectados por el escenario en el que ocurrieron, por ejemplo, en el campo, en la escuela, o en el hogar. En otras palabras, ellos estudiaron las características psicológicas de los eventos interpersonales particulares más que las relaciones entre persona, comportamiento y situaciones en un sentido más amplio. Ellos siguieron haciendo esto en estudios posteriores (e.g., Shoda y LeeTiernan, 2002). 


\section{Tabla 1}

Ejemplos de Escenarios Ecológicos, Situaciones Nominales, Situaciones Interpersonales y Características Psicológicas (de Shoda, Mischel y Whrigt, 1994).

\section{Escenario Situaciones nominales}

Trabajar la madera

\section{Campamento}

Escuela

Salón de clases

Hora de la comida

Hogar

Patio de juegos
Cuando el compañero inicia un contacto positivo

Cuando el compañero molesta, provoca o amenaza

Cuando se es elogiado por un adulto

Cuando se le llama la atención por un adulto

Cuando se es castigado por un adulto

Cuando el compañero inicia un contacto positivo

Patio de juegos

Reuniones de cabaña

Salón de clases

Hora de la comida

Mirando TV

Cuando el compañero inicia un contacto positivo

Cuando el compañero molesta, provoca o amenaza

Cuando se es elogiado por un adulto

Cuando se le llama la atención por un adulto

Cuando se es castigado por un adulto

Cuando el compañero inicia un contacto positivo

Cuando el compañero molesta, provoca o amenaza

Cuando se es elogiado por un adulto

Cuando se le llama la atención por un adulto

Cuando se es castigado por un adulto

Cuando el compañero inicia un contacto positivo

Cuando el compañero molesta, provoca o amenaza

Cuando se es elogiado por un adulto

Cuando se le llama la atención por un adulto

Cuando se es castigado por un adulto

Cuando el compañero inicia un contacto positivo

Cuando el compañero molesta, provoca o amenaza

Cuando se es elogiado por un adulto

Cuando se le llama la atención por un adulto

Cuando se es castigado por un adulto compañero, positivo

compañero, negativo adulto, positivo adulto, negativo adulto, negativo compañero, positivo compañero, negativo adulto, positivo adulto, negativo adulto, negativo compañero, positivo compañero, negativo adulto, positivo adulto, negativo adulto, negativo compañero, positivo compañero, negativo adulto, positivo adulto, negativo adulto, negativo compañero, positivo compañero, negativo adulto, positivo adulto, negativo adulto, negativo compañero, positivo compañero, negativo adulto, positivo adulto, negativo adulto, negativo 
El trabajo de Mischel y sus colegas es importante e interesante, pero sujeto a las limitaciones siguientes:

- Shoda y sus colegas redujeron "situaciones" a los comportamientos -en otros estudios (e.g., Shoda y LeeTiernan, 2002) incluso los rasgos- de una persona en esa situación.

- Ellos ignoraron la significancia psicológica de las cosas materiales y del arreglo material y social de las situaciones (Costall y Dreier, 2006).

- Ellos no estudiaron cómo los eventos y las relaciones interpersonales fueron afectadas por el escenario en el cual ocurrieron (Dreier, 2008a).

- Como muchos otros, Shoda y sus colegas se fijaron tanto en iluminar los factores de la personalidad que no estudiaron los factores situacionales y comportamentales; ni estudiaron el interjuego entre esos tres factores de una forma comprehensiva y minuciosa. En línea con su noción intuitiva de personalidad, en lugar de ello decidieron capturar las características duraderas, personales que subyacen a los patrones individuales de variación (Mischel, 2004, p. 1). Fleeson (2004) y Fleeson y Leicht (2006) muestran un interés similar.

- Cuando ellos estudiaron a una persona en más de una situación, analizaron cada situación por separado, como si no fueran afectadas por las otras situaciones en las cuales la persona también toma parte (ver también Funder, 2005; Wagerman y Funder, 2009).

Estudiar las relaciones entre persona, situación y comportamiento es de importancia fundamental para la psicología de la personalidad. Pero las maneras en las cuales la psicología de la personalidad define los ambientes y sus interacciones con las personas están marcadas por limitaciones importantes que han sido lamentadas por décadas (Funder, 2001; Lewis, 2002). Lo que se dice de una situación y sus vínculos con las personas y los comportamientos permanece en la vaguedad. Baumeister, Vohs y Funder (2007) de manera similar han señalado que la investigación de la personalidad también ha olvidado estudiar el comportamiento, incluso durante la "Década del Comportamiento" de la APA. La iluminación de la relación entre comportamiento, situación y persona es limitada y sesgada.

\subsection{Persona-situación-Comportamiento en LA VIDA COTIDIANA}

Algunos investigadores han sugerido compensar este desequilibrio e incrementar la fundamentación ecológica y la validez de la investigación en torno a la personalidad. Así, Mehl, Gosling y Pennebaker vieron "una necesidad urgente de la investigación observacional que vincule aspectos de las personalidades de la gente a sus comportamientos, ambientes e interacciones diarias" (2006, p. 864). En el más reciente manual Handbook of Research Methods in Persoanlity Psychology, Craik insistió en que necesitamos estudios de campo que revelen una personalidad "a través de las acciones situadas, diarias de los individuos" porque "A las personas se les conoce mediante su conducción y experiencias cotidianas" (2007, p. 211) y sus "vidas son vividas día a día, un día a la vez, de un día a otro, día tras día y de día en día" (Craik, 2000, p. 234; ver también Fleeson, 2004). Algunos, por ejemplo, Funder (2001, 2005; Wagerman y Funder, 2009) han usado métodos observacionales directos. Otros, como Craik (2000), usaron registros en vídeo. El surgimiento de nuevas tecnologías de la información ha promovido muchos métodos nuevos. La mayoría de estos incluyen muestreos temporales de comportamientos que ocurren naturalmente, captando a las personas por un período de tiempo mediante grabadoras de voz digitales, muy frecuentemente también obteniendo información sobre estados emocionales, experiencias y situaciones como en métodos de muestreo experiencial (Conner, Barrett, Tugade y Tennen, 2007; Fleeson, 2004; Fleeson y Leicht, 2006; Mehl et al., 2006). También hay un interés renovado en los métodos de diarios a solicitud para "captar la vida tal como es vivida" (Bolger, Davis y Rafaeli, 2003, p. 579).

Algunos de esos métodos adoptan una perspectiva de la tercera persona, otros una perspectiva de la primera persona. Algunos investigadores están principalmente interesados en la frecuencia de ocurrencia de actos o rasgos particulares (Craik, 2000; Mehl et al., 2006). Así, Fleeson (2004) encontró que la variabilidad de la manifestación de rasgos de la persona individual entre situaciones fue al menos tan grande como la variabilidad de rasgos entre personas. Otros investigadores están interesados en las emociones y sus variaciones a la hora de la excitación, y otros aún se enfocan en cuestiones concretas de la personalidad en relación al uso del tiempo cotidiano.

Ahora mencionaré brevemente un ejemplo de este conjunto de investigaciones, un estudio de Kahneman et al. (2004) quienes desarrollaron un "Método de Reconstrucción del Día". Comparado con el muestreo de experiencias, este es un método de investigación que consume menos tiempo y es menos perturbador de las actividades en curso de las personas estudiadas. Para lograr avances en la investigación sobre el recuerdo asistido, les pidieron a las personas escribir una narrativa breve del día anterior, dividirla en una secuencia de episodios y luego responder un número de preguntas sobre cada uno de los episodios 
en un formato de encuesta. Kahneman et al., estudiaron a 313 madres trabajadoras con este método y encontraron co-variaciones interesantes sobre el curso del día entre sus grados de energía/cansancio, sus estados emocionales y sus niveles de estrés, situaciones y actividades. Desde mi perspectiva, su hallazgo más relevante fue el profundo sentido de ambivalencia experimentado por estas madres trabajadoras sobre el regreso a casa después del trabajo. Por un lado, cuidar de sus niños fue lo de más importancia para ellas. Por el otro lado, ellas experimentaron un afecto negativo considerable dirigido a sus niños y a ellas mismas en ese momento del día.

En relación al desarrollo de teoría, podemos aprender lo siguientes de este estudio:

- Kahneman et al. (2004) estudiaron persona-comportamiento-situaciones en una secuencia temporal y no en situaciones aisladas.

- Esto les permitió captar el hecho de que el impacto de una situación sobre la persona se vio afectado por su localización en la secuencia de situaciones y actividades a lo largo del día.

- Así, las variaciones en los estados emocionales generales simplemente no siguen curvas diurnas de cansancio.

- Pero Kahneman et al. dieron por sentado el curso del día. Lo trataron como si fuese una unidad natural de actividad.

- No consideraron el impacto que tienen los arreglos sociales sobre cómo las personas viven sus vidas diarias.

\section{Una Propuesta Teórica}

Ahora delinearé algunos conceptos básicos en una teoría de las personas que va más allá de las limitaciones en la investigación sobre la personalidad resumida en la primera parte de este escrito. Esta teoría descansa en una aproximación comprehensiva a persona-situación-actividad. Nos puede guiar al estudiar a las personas en y a través de varias situaciones. Considera las situaciones como partes de los contextos y prácticas sociales en las cuales toma lugar. Las personas son vistas como existiendo en movimiento a través del tiempo y de los contextos. Esto nos permite captar cómo el orden de las vidas cotidianas afecta el funcionamiento de las personas. Otras teorías también pueden desarrollarse para resolver las limitaciones en la investigación de la personalidad mencionadas anteriormente. La presente teoría tiene la ventaja de fortalecer la validez ecológica de teorizar y de los hallazgos empíricos al aproximarse a la personalidad desde la perspectiva de la vida cotidiana de las personas. También puede desarrollar nuestro entendimiento de las intervenciones psicológicas, tal como veremos en la tercera parte. La teoría fue desarrollada como parte de mis propios estudios teóricamente motivados sobre intervenciones psicológicas en relación a la psicoterapia (Dreier, 2008a) y el aprendizaje (Dreier, 2008b). También está inspirada en estudios similares de intervenciones en otro campo de práctica realizados por miembros de mi grupo de investigación sobre la "conducción personal de la vida cotidiana e intervenciones". La presentación de la teoría se ilustrará brevemente con hallazgos de esta investigación. Cuando no se den referencias específicas, la presentación está basada en material de Dreier (2008a).

\subsection{Orden y arreglos}

Las personas viven en sociedades con un cierto orden social. Una característica importante de un orden social es el arreglo de las prácticas sociales a través del cual las sociedades y las vidas de las personas son re-producidas y cambiadas (Schatzki, 2002). Una característica de estos arreglos sociales es usualmente minimizada en la psicología a pesar de que es importante para la vida de las personas. Las sociedades están divididas en diversos contextos sociales tales como lugares de trabajo, hogares, escuelas, etc. Estos contextos sociales son unidades socio-materiales para prácticas sociales. Frecuentemente están institucionalizados y constituyen las dimensiones espaciales de la vida cotidiana. Un contexto social es un lugar para llevar a cabo todas, o partes particulares de, una o varias prácticas sociales tales como el trabajo, la educación, el cuidado de la salud, la crianza infantil y cocinar. Un contexto social por lo tanto implica demandas y responsabilidades particulares para las personas que participan en su(s) práctica(s) social(es). Los contextos sociales están separados uno de otro, pero también están vinculados con otros contextos sociales en formas particulares que canalizan cómo las prácticas sociales pueden llevarse a cabo entre ellos. Además, el arreglo de los contextos sociales a menudo define quién cuenta como un participante legítimo en ellos, debido a que la persona particular ocupa un lugar como propietario, empleado, por competencias, membresía y parentesco. El arreglo de un contexto social también involucra usualmente posiciones sociales particulares en las cuales personas particulares toman parte, en formas diferentes. Incluso algunos contextos sociales incluyen arreglos para cuando una persona puede cambiar a otra posición en ese contexto o de otra manera llegar a participar en otras partes de sus prácticas sociales sobre el curso del tiempo.

Por ejemplo, piénsese en las escuelas con clases graduadas en función de la edad, los sujetos escolares, el momento del calendario escolar, los recreos y los programas como un arreglo institucional que da forma y afecta al curso y la dinámica de la participación y el 
aprendizaje (Dreier, 2008b). Mientras las prácticas educativas son principalmente llevadas a cabo en las escuelas, dependen de otros contextos sociales de la vida de los niños y están dirigidas a desarrollar competencias para la participación de los niños en otra parte.

Además, un contexto social pequeño puede estar comprendido en un contexto social mayor. Un salón de clases, por ejemplo, está arreglado de una manera particular para llevar a cabo partes particulares de las prácticas educativas en el contexto mayor que es una escuela (Sørensen, 2009). También hay otras clases de arreglos sociales, tales como las legislaciones, recomendaciones, reglas y agendas.

Las actividades de las personas y las relaciones entre personas son parte de las prácticas sociales que toman lugar en contextos sociales particulares y se ven afectados por sus arreglos sociales. Así, algunas actividades y relaciones de una persona son parte de las prácticas y arreglos familiares, mientras que otras actividades y relaciones son parte de, digamos, las prácticas laborales de la persona. Las personas asocian diferentes intereses, propósitos e historias con esas diferentes actividades, relaciones, prácticas y contextos. El significado y el curso de eventos y situaciones particulares y delimitadas también se ven afectados por el contexto y los arreglos en los cuales ocurren.

Finalmente, hay arreglos sociales de cómo llegamos a vivir nuestra vida cotidiana. Primero que todo, esos arreglos sociales establecen un ordenamiento de cuándo podemos o debemos participar en contextos sociales particulares. Piénsese en los arreglos sociales ordinarios que estructuran nuestras mañanas cotidianas, las horas de trabajo, las horas en las que empezamos, las tardes y las noches en las cuales organizamos nuestros alimentos y otros momentos de descanso dispersos a través de ellos. Piénsese también de los días laborales, los fines de semana y las vacaciones. Esos arreglos sociales introducen ciertos ritmos de actividad y ciertos cambios, rupturas y tensiones internas en el curso de nuestras actividades cotidianas. Así, la vida familiar en un día se divide en una pequeña parte temprano por la mañana y otra por la tarde, con mucho más sucediendo en otras partes en el medio. Los miembros de la familia pasan tiempo diferencialmente en la casa, en el trabajo o en la escuela. Mucho más se podría decir de los arreglos sociales de las prácticas, pero esto es suficiente para recordarnos que logramos una psicología más rica y más apegada al mundo al incluirlos en nuestro estudio de las personas.

\subsection{Participación situada y movimiento}

Como otros investigadores con aproximaciones similares (e.g. Harré, 1998; McAdams, 2006), yo prefiero el término persona para denotar una aproximación ho- lística y del agente para el estudio de la personalidad. Como seres de carne y hueso, las personas siempre están situadas en una ubicación desde donde sus perspectivas sobre su experiencia y sus actividades se extienden hacia el mundo. Es más, sus actividades y experiencias son parte de sus relaciones con otros de quienes dependen y con quienes comparten tales prácticas sociales. Se coordinan juntos directamente en el contexto social en el cual estas personas se localizan, así como indirectamente mediante las secuencias de actividades, las diferentes tecnologías, el habla y las reflexiones que vinculan lo que hacen en este momento aquí y ahora con otros momentos y lugares. Las personas son agentes quienes deben desarrollar habilidades para tomar parte en arreglos y prácticas sociales complejos y para afectarlos. La agencia de una persona está tan profundamente entrelazada con las prácticas sociales en las que vive, que es más adecuado hablar acerca de las personas como participantes y sobre participación más que de agencia y actividad, acción o comportamiento. Los contextos sociales particulares tales como la familia de una persona contienen relaciones particulares, co-participantes y alcances de posibilidades para participar. Las personas asocian preocupaciones particulares con contextos sociales particulares y tienen cosas particulares pendientes que hacer en ellos. Los participantes ponen atención a cosas particulares, tienen experiencias particulares, tienen y provocan estados mentales particulares y reflexionan sobre sus vidas de maneras particulares. Pero como las personas se mueven hacia otros contextos sociales, tales como sus escuelas o sus lugares de trabajo, encuentran otros arreglos, posiciones, relaciones y co-participantes, en donde tienen otras preocupaciones y otras cosas pendientes, y así su participación adquiere otros significados. Ellos ponen atención a otras cosas, tienen otras experiencias, están en otros estados mentales particulares, y reflexionan diferencialmente sobre sus vidas. En pocas palabras, el funcionamiento psicológico de las personas tiene cualidades y dinámicas situadas, y varía en cuanto las personas se mueven de un contexto a otro. Esto nos debería prevenir contra las evaluaciones sobre-generalizadoras de las personas basadas en su conducción en un contexto social.

Ordinariamente, las personas viven vidas cotidianas las cuales se extienden a través de diversos contextos sociales. Se mueven a través de y participan en varios contextos. Las personas participan en algunos contextos sociales diariamente, en otros regularmente, y aún en otros ocasionalmente o solo una vez. Después de largos o cortos periodos de sus vidas, la combinación de contextos sociales y su significado para las personas puede cambiar. Usualmente 
las personas ven una vida con muchos ángulos con participación en una variedad de contextos sociales que les ofrecen una variedad de relaciones, actividades y experiencias enriquecedoras. Esto se puede reducir para algunas personas que son excluidas de ciertos contextos sociales, por ejemplo, debido a su desempleo o a su incapacidad. La complejidad de las vidas de las personas y las diversas demandas hechas a las personas debido a su participación en diversos contextos sociales implica una necesidad correspondiente para desarrollar diversas destrezas, competencias y entendimientos personales.

En sus complejas vidas cotidianas, las personas persiguen muchas preocupaciones a través de varios contextos sociales. Al hacerlo, ellas toman en cuenta que diferentes contextos sociales ofrecen diferentes relaciones y posibilidades para resolver o responder a esas preocupaciones. Ser bueno para dirigir sus propias persecuciones a través de contextos particulares involucra ser experto en relación a los arreglos sociales. Como veremos en la tercera parte, las personas comparan similitudes y diferencias entre sus experiencias y resultados en contextos sociales diferentes y toman ventaja de esto en la persecución de sus complejos cursos de cambio y aprendizaje (Dreier, 2008b).

Las personas también arreglan los contextos sociales ordinarios de sus vidas cotidianas para ajustar sus prácticas preferidas. Arreglan sus cuartos en sus casas para ajustar sus prácticas preferidas, digamos, sus cocinas para ajustar sus prácticas de cocinar, por ejemplo, re-arreglar sus estantes de las especias cuando cambian sus preferencias de comidas (de Léon, 2006). Estos son arreglos personales en relación a arreglos socio-materiales existentes de, digamos, sus departamentos. Los arreglos personales a menudo descansan en arreglos y entendimientos entre participantes acerca de sus prácticas preferidas. Ellas cambian estos arreglos para acomodarse a los cambios en sus preferencias. Las personas también arreglan los diferentes contextos sociales de acuerdo a sus preferencias situadas. Gosling et al. (2002) encontraron que las personas arreglan sus oficinas y sus dormitorios y que podemos leer sus personalidades muy bien al tomar en cuenta esos arreglos como señales.

\subsection{Conducción de la vida cotidiana}

Además, debido a la complejidad de las vidas cotidianas, conducir una vida cotidiana se ha convertido en una necesidad. Las personas se ven obligadas a cubrir sus necesidades y hacer lo que es más importante para ellas. Deben llevar a cabo sus vidas cotidianas consistentemente de manera que no se desmoronen debido a sus diversas actividades y compromisos en muchos contextos sociales y relaciones. No basta con simplemente afrontar cada demanda y situación individual. Deben hacer frente a vivir una vida cotidiana compleja como un todo. Una conducción personal de vida, entonces, no es simplemente cuestión de gastar el tiempo, como es visto en los estudios del uso del tiempo, o simplemente un estilo de vida, como una forma característica de vida individual. Es algo que una persona debe lograr ( $\mathrm{Hol}-$ zkamp, 1995, 1996). Al hacerlo, las personas pueden tener éxito o fracasar, más o menos, con respecto a asegurar las demandas y preferencias personales. Las personas tienen cuidado de sí mismas mediante su conducción de la vida cotidiana en relación a sus arreglos sociales. Esta es una labor profundamente personal. Weber, quien introdujo el término hace un siglo en su estudio de La Ética Protestante y el Espíritu del Capitalismo (1952), enfatizó el rol de los valores religiosos secularizados en la conducción de la vida cotidiana de los primeros grupos protestantes que él estudió. Los valores religiosos sin duda pueden jugar un rol en la conducción de la vida cotidiana. Pero, a un nivel más básico, una conducción de la vida cotidiana es un arreglo personal en relación a los arreglos sociales de la vida cotidiana.

Una conducta de vida cotidiana involucra a las personas coordinando sus diversas obligaciones, relaciones y actividades con su diferentes co-participantes en diversos contextos sociales a lo largo del día. Al hacerlo, las personas construyen agendas más o menos holgadas y desarrollan secuencias diarias de actividades más o menos elaboradoras para llevarse a cabo regularmente. Por ejemplo, la mañana en muchas familias está compuesta por rutinas que coordinan y aceleran ciertas actividades y relaciones. Mientras las rutinas o hábitos aseguran que las personas consigan hacer lo que necesitan hacer, y se consigan más tiempo para otros asuntos importantes, también introducen un grado de ordinariedad en las vidas cotidianas. Además, las rutinas o hábitos son sedimentaciones familiares e íntimas de una forma preferida de conducir la vida propia. Por lo tanto, pueden sostener un profundo significado personal, incluso aunque este significado a menudo se dé por garantizado. Holzkamp (1995) apunta esto en el caso de una persona que vuelve a su casa después de haber estado en un hospital y es impactado por el profundo significado personal de muchas de sus rutinas hogareñas. En realidad, los hábitos descansan sobre preferencias, y pueden cambiarse al cambiar las preferencias si la situación y los co-participantes con uno lo permiten.

Pero al buscar generalidades en el nombre de la ciencia, debemos ser cuidadosos de no sobreestimar el grado de regularidad en las vidas cotidianas. Las personas también atesoran, cultivan y persiguen variaciones y descansos en sus vidas cotidianas. Demasiada regularidad hace 
que la vida de uno se vuelva muy monótona, ordinaria y aburrida. Es más, hay diferencias individuales en las clases y grados de ordinariedad y variación que las personas prefieren en sus vidas cotidianas. Se manifiestan en diferencias individuales en las maneras en las que las personas prefieren conducir sus vidas cotidianas. Estas diferencias individuales son el caldo de cultivo de muchos conflictos entre personas que viven en contacto estrecho.

Las personas negocian la conducción de sus vidas cotidianas con muchos otros con quienes comparten parte de ella en varios contextos sociales, así con la esperanza de lograr un balance deseado y necesariamente personal de actividades y compromisos a través de los contextos y los días. Pero una parte particular compartida de la vida cotidiana, tal como la vida familiar, tiene diferentes significados para sus miembros individuales cuyas vidas cotidianas involucran otros contextos sociales que no son compartidos. Al negociar y arreglar sus vidas familiares compartidas, los miembros deben tomar en consideración las diferentes composiciones trans-contextuales de sus vidas cotidianas y los diferentes significados individuales de su vida familiar. Esta es una razón por la cual los arreglos de una parte compartida de la vida pueden variar. Los arreglos pueden ser más o menos fijos y detallados o más o menos holgados y abiertos. Los arreglos familiares holgados y abiertos hacen más fácil valorar las oportunidades que surgen, como cuando los amigos de los niños les invitan a compartir sus actividades. Y los arreglos familiares más fijos y detallados hacen que las responsabilidades de los muchos quehaceres en la conducción de la vida cotidiana de la familia se realicen. Las personas individuales desde luego preferirán diferentes tipos y grados de arreglos en la conducción de sus vidas cotidianas. Las diferencias y conflictos de edad y género fluyen a partir de los tipos y grados preferidos de arreglos familiares compartidos.

Al establecer una conducción de la vida cotidiana, la vida de una persona se vuelve marcada por sus "compromisos con otras personas específicas en ricas y concretas relaciones sociales, con lugares específicos y sentidos de lugar, con actividades específicas y organizaciones de ritmos de vida" (Smith, 1987). El auto-entendimiento que una persona desarrolla está en estrecha vinculación a su conducción de la vida cotidiana. Es un entendimiento de sí mismo como una persona con ciertos compromisos que conducen su vida en un cierto camino, valorando ciertas oportunidades, respondiendo a situaciones, desafíos y contratiempos, prefiriendo ciertas actividades, ritmos y relaciones, etc. En otras palabras, el auto-entendimiento de una persona emerge de su conducción de la vida cotidiana y la guía (Holzkamp,
1995). No consiste en un conjunto de atributos descontextualizados, sino que se desarrolla a lo largo del desarrollo de la conducción de vida de una persona.

El desarrollo de una conducción personal de la vida cotidiana involucra el aprendizaje de destrezas y entendimientos. Algunas de esas destrezas y entendimientos tienen que ver con cómo las personas se conducen en las transiciones en relación a cambios y rupturas en sus actividades cotidianas, relaciones, situaciones y contextos sociales. Esta es la razón de la ambivalencia emocional de las madres que vuelven a sus casas después del trabajo en el estudio de Kahneman et al. (2004). En el centro de este asunto yace la relación entre los arreglos sociales de las vidas cotidianas, la distribución de responsabilidades y las maneras en las cuales las madres, los niños y los padres se mueven a lo largo de las transiciones y el arreglo de la vida de trabajo/hogar en el curso de las tardes familiares.

En cuanto la vida de la gente cambia, debe cambiar su conducción de la vida cotidiana y sus destrezas y entendimientos asociados. Tales cambios pueden ser particularmente intensos y complicados cuando las personas son afectadas por eventos inesperados y perturbadores, tales como cuando un cónyuge es diagnosticado con un tumor cerebral (Madsen, 2011), o a una mujer le es detectado un tumor cancerígeno asintomático (Ryle, 2010) o una prueba genética revela la enfermedad de Huntington en una familia en riesgo (Huniche, 2009). Pero todas las personas cambian su conducción de la vida cotidiana muchas veces y en muchas formas a lo largo de su vida. Es también particularmente obvio en el curso de la niñez (Dreier, 2009b). Los arreglos sociales para el desarrollo del niño involucran cambios frecuentes en su participación en instituciones grandes, y cambios de una institución a otra, que se distinguen por diferentes demandas y compromisos. Esto conduce a cambios frecuentes en la composición y el balance de las vidas cotidianas complejas de los niños y en sus actividades y relaciones. Durante el curso de esto, y en cuanto un niño se vuelve menos dependiente de los adultos y los arreglos institucionales de cuidado, ellos gradualmente deben desarrollar una conducción de ña vida cotidiana, su propia forma de tener cuidado de sí mismos en práctica sociales con las destrezas y auto-entendimientos necesarios.

En este punto termino de delinear una teoría de las personas basada en su conducción de la vida cotidiana. Esta teoría puede enriquecer nuestro entendimiento de la personalidad humana al dirigir nuestra atención hacia el interjuego de la vida real entre persona, situación y actividad y hacia cómo las personas encuentran y dirigen las situaciones de la vida real. Desde luego que esto puede 
promover la validez ecológica de los hallazgos empíricos y la teorización en la investigación sobre la personalidad.

\section{IMPLICACIONES PARA ENTENDER LAS INTER- VENCIONES PSICOLÓGICAS}

Esta sección ilustra cómo esta aproximación teórica sobre las personas puede provocar y enriquecer nuestro entendimiento de las intervenciones psicológicas.

\subsection{Arreglo de intervenciones a través de contextos}

En general, las prácticas expertas son arregladas de manera tal que toman lugar en un contexto social particular el cual se aparta o está aislado de las vidas cotidianas ordinarias de las personas a las que sirven. La educación se lleva a cabo en salones de clases, la terapia en las sesiones, el cuidado de la salud en consultorios u hospitales, etc. Aun así, estas prácticas expertas están destinadas a tener un efecto benéfico en las vidas cotidianas de las personas. No obstante, las teorías y la investigación sobre esas prácticas son sorprendentemente ciegas al impacto de este arreglo de aislamiento en el curso y la dinámica de los procesos que pretenden fomentar. Uno podría asumir que este arreglo descansa en experiencias históricas acerca sobre esto como la mejor manera de promover los efectos de intervenciones sobre las vidas cotidianas de las personas a las que sirve. Y sería razonable esperar explicaciones claras de los efectos que provocan esas prácticas aisladas al impactar sobre las vidas cotidianas de las personas a las que sirve, así como de las dificultades que ese arreglo crea para tener un efecto. Pero tales explicaciones son gravemente ausentes. En general, la investigación asume que los efectos de esas prácticas expertas se llevan a cabo en la situación inmediata de la intervención en los salones de clase, las sesiones, las consultas, etc. y después simplemente transferidas a la vida cotidiana y aplicadas por pacientes y pupilos dóciles. Esta explicación viene de una noción en la que el aprendizaje se entiende como un proceso de transmisión-internalización-transferencia-aplicación (Dreier, 2008b; Lave, 2011). Yo he trabajado por años en alcanzar un entendimiento más claro del inter-juego entre las sesiones de terapia y las vidas cotidianas de los clientes. Cuando empecé el proyecto ahora publicado en el libro Psychoterapy in Everyday Life (2008a), pronto se volvió claro que este entendimiento general no correspondía con lo que realmente hace el trabajo de terapia en la vida cotidiana de los clientes. Aparte de estudiar a las personas cambiando en sus vidas cotidianas, este proyecto también pretendía generar un entendimiento más adecuado sobre lo que está implicado en hacer trabajo de intervenciones.
El proyecto se llevó a cabo en una unidad externa de psiquiatría infantil en Copenhague. Involucró un número pequeño de terapias familiares a largo plazo conmigo como co-terapeuta. Todas las sesiones fueron grabadas en audio y un asistente de investigación entrevistó a las familias en sus hogares en intervalos regulares a lo largo del período de su terapia y hasta medio año después de su terminación. En las entrevistas a los clientes primero se les preguntó sobre sus vidas cotidianas: ¿Qué sucedió en sus vidas cotidianas desde la entrevista anterior? ¿Se produjeron algunos cambios? ¿Cuáles y cómo? ¿Qué rol jugaron en esos cambios? ¿Hicieron cosas de forma diferente que antes? ¿Sus sesiones influenciaron estos cambios? Y de ser así, ¿Cómo? Sólo después de esas cuestiones, se les preguntó sobre sus experiencias de sus sesiones, sus terapeutas y su participación en las sesiones. Este diseño reflejó un entendimiento descentrado de la terapia. La terapia se vio como fluyendo de la vida cotidiana de los clientes. Las sesiones fueron vistas como partes particulares de sus vidas cotidianas en curso más que aquello de lo cual fluye el efecto de la terapia. El libro (Dreier, 2008a) contiene una presentación elaborada de la teoría de las personas y un análisis detallado del inter-juego entre las sesiones y las vidas cotidianas de una familia y sus miembros a lo largo de un período de año y medio. Mencionaré unos cuantos puntos principales de este análisis.

\subsection{Búsqueda de cambios a través de contextos}

Los clientes deber buscar los cambios a sus problemas a través de los contextos sociales porque las sesiones terapéuticas ocurren en un lugar aislado fuera de sus vidas cotidianas ordinarias mientras que sus problemas son partes de sus vidas cotidianas en varios contextos sociales. Deben aprender a buscar una solución a sus problemas en sus sesiones y fuera de ellas de nuevo, dentro y a través de los contextos sociales cotidianos de su hogar, su escuela, trabajo, etc. Para hacerlo bien, deben hacer algo diferente en torno de sus problemas en esos contextos sociales y aprender a combinar las diferentes cosas que hacen de una manera coherente.

Por ejemplo, la hija menor de doce años en el caso de la terapia familiar (Dreier, 2008a) sufría de ataques de ansiedad. En los distintos contextos de su vida cotidiana, estos ataques tenían diferentes significados para ella y tenía diferentes posibilidades para hacer algo por resolverlos. Sus motivos para hacer algo sobre sus ataques, y sus motivos en relación a lo que tenía que hacer sobre ellos difería a través de los contextos. En su casa, sus padres le ayudaban y confortaban. Ella dependía de que ellos hicieran algo y no creía que lo necesitara, y fue capaz de hacer algo 
con su ansiedad con su propio acuerdo. En la escuela, sin embargo, tenía miedo de ser ridiculizada, que se burlaran de ella y de perder a sus amigos si se enteraban de su ansiedad. Esto creó muchas dificultades referentes a cómo ella manejaba su vida en la escuela. Esto convirtió el superar los ataques importante y difícil al mismo tiempo. En las sesiones ella valoraba tener oportunidad para hablar con un adulto quien estaba interesado en ella, pero nunca había entendido precisamente cómo el hablar sobre sus ansiedades podría ser un medio para superarlas. No obstante, si la terapia tuvo que ver en la solución de sus problemas, fue porque ella tuvo que aprender a combinar los diferentes significados de y posibilidades para hacer algo en relación a sus ansiedades en una compleja y variada búsqueda de cambios a través de sus varios contextos sociales.

Aunque los clientes buscaron cambios a través de las sesiones y los varios contextos sociales de sus vidas cotidianas, ellos enfatizaron que las sesiones eran completamente diferentes para ellos comparadas con los otros contextos sociales de sus vidas cotidianas. Para ellos, las sesiones ofrecían una extraña intimidad, que difería de sus intimidades con la familia y los amigos, entre otras cosas, porque sus terapeutas eran una especie de extraños íntimos para ellos. Ellos tenían que volverse familiares con ellos y hacer uso de la forma peculiar de práctica en las sesiones. Pero empáticamente insistieron que en casa nunca habían hablado entre ellos como lo hicieron en la conversación sobre problemas concertada de las sesiones de terapia. Nunca transfirieron y aplicaron esta forma de conversación a sus conversaciones en casa. No podían y no lo harían por muchas razones. De hecho, parecía que las sesiones hacían una diferencia para ellos precisamente porque las sesiones eran diferentes.

Los clientes usaron las ideas de la sesión y las recomendaciones de maneras situadas en las diversas situaciones y contextos sociales de sus vidas cotidianas con varias cosas en juego para ellos. Ellos ajustaron estas ideas y recomendaciones con sus habilidades y sus diversas posibilidades en relación a los diversos co-participantes en sus contextos sociales. Variaba cómo una idea o parte de una recomendación de las sesiones fue usada y lo que se necesitaba para usarla. Por ejemplo, provocado por los problemas de su familia y las pláticas acerca de ellos en las sesiones, el padre en este caso se dio cuenta que se sentía presionado y le resultó difícil identificar y mantener su postura. Él veía que esto era un denominador común de sus problemas en el hogar, en las sesiones de terapia con su familia y sus terapeutas y en su lugar de trabajo en relación con sus colegas y jefes. Pero lo que le llevó a clarificar sus posturas y a sostenerlas fue completamente diferente en estos diversos contextos sociales. Al comparar esos asuntos y oportunidades en los diferentes contextos sociales, encontró maneras diferentes de buscar este rasgo común de sus problemas en sus diferentes contextos sociales.

Usar las ideas y las recomendaciones de la sesión adecuadamente a menudo implica aprendizaje, y las ideas y las recomendaciones a menudo tienen que transformarse para volverse usables. En otro lugar y después los clientes modificaron y cambiaron las ideas y las recomendaciones de las sesiones, a veces de maneras sorprendentes, y de formas sobre las cuales los terapeutas raramente se enteraban. Por ejemplo, en la casa después de una sesión de terapia, los padres reinterpretaban las preguntas de sus terapeutas sobre los problemas recurrentes entre sus dos hijas en la mañana como parte de una recomendación para ellos que como padres no podían tolerar el problema que causaban sus niñas. Esta era una interpretación radical de lo que los terapeutas habían tenido en mente, así como una forma radical de cómo estos padres usualmente habían reaccionado a los problemas de sus niñas. Por algún tiempo, esto convirtió la cuestión de estar enfadado, y de quien estaba realmente enfadado, en uno de los principales asuntos en casa y en las sesiones. Pero este asunto fue también transformado posteriormente. De hecho, los clientes continuaban modificando las ideas y recomendaciones de las sesiones debido a que sus vidas en curso continuaban cambiando. Los cambios en muchos de los problemas relacionados con la terapia no tienen, de hecho, límites fijos. No tienen un punto final definido o solución, desde cuando los clientes pueden olvidarse de todo acerca de ellos definitivamente y no necesitan preocuparse por ello por más tiempo. Esto ocurre porque los clientes a menudo están inicialmente confundidos o indecisos acerca de sus problemas y qué hacer con ellos y pasan por procesos tediosamente largos, en donde reconsideran y cambian sus opiniones sobre dónde están parados y lo que quieren hacer de sus problemas y sus vidas en curso. Cambios en los problemas y en sus auto-entendimientos van de la mano con cambios más comprehensivos en las vidas cotidianas de los clientes. Los procesos a largo plazo del padre descubrieron su postura y cómo se aferraba a esta, lo cual no es sino un ejemplo de esto. 


\subsection{Intervenciones en las vidas cotidianas}

Todo esto indica que una intervención no trabaja como un efecto de lo que hace un experto de manera que el resultado de la terapia se pueda referir al experto y sus procedimientos. Lo que sucede está en relación estrecha al significado literal de la palabra intervenir como

"(1) Llegar, ser o yacer entre, (2) tomar lugar entre dos eventos, puntos del tiempo, etc., (3) llegar o estar entre como algo innecesario o irrelevante, (4) venir entre como una influencia, como con el fin de modificar, asentar o impedir alguna acción, argumento, etc." (Webster's New World College Dictionary, 1997, p. 707).

Una intervención ocurre entre todo lo que ya está pasando. La investigación en psicoterapia debe reconocer que la terapia nunca trabaja sola. Siempre trabaja a lo largo de y en interacción con otras influencias del ambiente, y las experiencias y respuestas del cliente en relación a estas influencias. Cómo trabaje, depende de otras circunstancias y eventos, así como de las respuestas e iniciativas del cliente y de las respuestas e iniciativas de otras personas en sus vidas diarias en curso. Por ejemplo, sólo cuando emergió una oportunidad inesperada para su actividad muy querida de montar a caballo con un amigo lejos del hogar hizo que la hija más joven empezara una persecución intensa y rápida para superar sus ansiedades dentro y fuera de las sesiones. En realidad, las vidas cotidianas de los clientes pueden ser cambiantes por muchas razones, que afectan la dinámica y el curso de su terapia en curso (ver también MackriII, 2008a). Es la razón por la cual necesitamos conocer cómo la terapéutica y otras influencias interactúan y son combinadas por los clientes en sus vidas cotidianas.

En las vidas cotidianas de los clientes ocurren muchas cosas al mismo tiempo y compiten por su tiempo y atención. Las preocupaciones relacionadas con la terapia son igualmente perseguidas en medio de muchas más. No se mantienen continuamente en sus mentes, sino que se ven interrumpidos por otros asuntos más importantes o no tan importantes. Los procesos de cambio relacionados a la terapia son discontinuos, o más aún difusos. Tienen que retomarse nuevamente para ser continuos, si no, corren el riesgo de olvidarse o perderse. Puesto que las ocasiones para retomarlos no se pueden predecir, los clientes deben aprender a darles su lugar sobre la marcha. A menudo además se les debe negociar con otros, quienes están en la disposición de hacer su parte, cuando están de acuerdo en que se les retomen.

\subsection{Intervenciones y conducción de la vida cotidiana}

En términos generales, como las intervenciones trabajan en las vidas cotidianas de los clientes depende de su conducción de la vida cotidiana. Así, es importante para los clientes, y para quienes comparten su vida con ellos, que al direccionar los problemas no dejen a la deriva, perturben o ensombrezcan las cosas que ellos valoran más y todas las otras cosas que deben de hacer en sus vidas cotidianas. En este sentido, direccionar los problemas debe ajustarse en su conducción de la vida cotidiana. Si una idea o una pare de recomendación no se ajustan a la conducción de la persona de su vida, probablemente no se usará o se dejará una vez más. En otras palabras, lo que se use de las sesiones dependerá de cómo se ajuste en las vidas cotidianas de los clientes. Lo que se llama una recaída, a menudo consiste en volver a caer en una conducción de la vida cotidiana precedente que el cliente no tuvo mucho éxito en cambiar. Una intervención es algo extraordinario que debe hacerse parte de la vida ordinaria con el fin de que se sostenga y de que no se olvide una vez más después de cierto tiempo.

Una conducción de la vida cotidiana existente a veces necesita ser cambiada mediante intervenciones para que pueda trabajar adecuadamente. Los clientes entonces pueden cambiar su conducción de la vida cotidiana con el fin de resolver sus problemas presentes. Reconocemos esto desde las intervenciones sobre el cuidado de la salud en las así llamadas enfermedades de estilo de vida. En terapias de familia, como en el caso de arriba, los padres pueden sorprenderse al darse cuenta de que la manera en que cuidan de sus niños hace a sus niños demasiado dependientes de ellos y restringe su desarrollo. Esta cuestión de amor y cuidado se vincula estrechamente con qué tan a menudo especialmente la madre distribuye y prioriza sus compromisos entre su familia y otros contextos sociales. Esto infunde su conducción de la vida cotidiana y su auto-entendimiento. Cambios en su conducción de la vida cotidiana promueve entonces cambios en las maneras en las cuales sus niños abordan sus problemas.

Así, los clientes a veces cambian su conducción de la vida cotidiana con el fin de compensar lo que ven como una negligencia o error anterior. Esto pasó con la hija mayor en el caso de arriba. En años anteriores, ella había expandido sus actividades y relaciones en otros contextos sociales fuera de la familia. Pero ella se vio envuelta en serios problemas con sus amigos. Dichos problemas normalmente ocurrieron fuera de su familia, pero también ocurrieron cuando ella invitó a sus amigos a su casa cuando sus padres no estaban. Las cosas se salieron tanto de sus manos que el propietario del complejo 
de apartamentos amenazó a la familia con desalojarlos. Ella reaccionó con fuertes sentimientos de vergüenza y tenía muchas ganas de volver a su anterior conducción de vida para restaurar sus relaciones familiares y mejorar sus relaciones entre su vida dentro y fuera de la familia. Los niños típicamente expanden sus actividades y relaciones fuera de la familia en sus años tempranos en la escuela. Esto cambia el balance en la conducción de la vida cotidiana de los niños, promueve exploraciones de nuevas actividades y lleva a nuevas amistades más o menos prolongadas en las relaciones con sus pares con nuevas confidencias e intimidades que se separan de la supervisión estrecha de sus padres. Los padres a menudo están inciertos sobre lo que sucede y cómo sus niños están llevando a cabo sus relaciones y actividades de las cuales no llegan a saber mucho. Casi de forma constante deben practicar formas indirectas de cuidado de sus niños fuera del hogar. Si sus niños llegan a tener un problema en sus relaciones y actividades en otros lados, el cuidado de los padres es enterrado fácilmente detrás de sus preocupaciones. La hija mayor en el caso de arriba estaba pasando por cambios tan rápidos en la conducción de su vida cotidiana que su auto-entendimiento difícilmente podía coincidir con los cambios en curso. En ciertos aspectos, su auto-entendimiento la sobrepasaba. Ella se enfocaba en sus esperanzas para el futuro, pero tomaba mucho de lo que esperaba ser capaz de lograr como algo de lo que ya era capaz y para lo que tenía derecho. En otras ocasiones, su auto-entendimiento se le rezagaba como si ella no fuera plenamente consciente de algunas de las habilidades que ya había desarrollado. El asunto de lo que constituye un auto-entendimiento realista -O, como se veía desde la perspectiva de sus padres, su reconocimiento de la persona en la que se había convertido- por supuesto que era precario. Choques entre incertidumbre, reconocimiento, confianza y desconfianza ocurrieron mientras sus padres no compartían lo que ella pensaba acerca de la persona que ella misma creía ser y lo que creía acerca de lo que era capaz, y lo que tenía derecho, de hacer. En los cambios en curso ocurriendo en la conducción de la vida de la hija más joven, explorar actividades y relaciones con sus amigos significaban más para ella, mientras que ella asumía que sus compromisos con la familia podían esperar y no creía que en su casa fuese irritable, porque, como ella lo veía, ella estaba aburrida en su casa, especialmente cuando no tenía la compañía de sus amigos. Las dos hijas mostraron una dinámica completamente diferente y tuvieron una ubicación primaria de sus compromisos que lo que la madre hacía en su conducción de vida centrada más en la familia. Por supuesto que ellos hicieron un uso muy diferente de las intervenciones de la terapia familiar.

Otro proyecto de mi grupo de investigación también dio luz sobre las relaciones entre los problemas de los clientes y su conducción de la vida cotidiana. Mackrill (2007, 2008a, 2008b, 2008c, 2011) usó diarios solicitados para darle seguimiento a las relaciones entre las sesiones de consejería y las vidas cotidianas de los adultos jóvenes que crecían en familias alcohólicas. Al intentar resolver sus problemas, estas personas jóvenes se basaron en muchas fuentes diversas. Hubo claras indicaciones de que sus problemas se intensificaron y que acudieron a la consejería psicológica cuando habían dejado el hogar y encontraron difícil llegar a comportarse como adultos en sus vidas cotidianas con sus propias parejas, ya fueran novios o novias.

\section{ConCLusión}

Debería volverse obvio ahora que la teoría de las personas que yo he delineado se inspira profundamente en el estudio empírico de las vidas cotidianas de las personas quienes están sujetas a intervenciones expertas. Las cuestiones de la práctica psicológica me recuerdan la relevancia de esta aproximación teórica y guían la dirección de su desarrollo. La teoría de la persona descrita arriba se basa en proyectos de investigación práctica empírica que tiene la ventaja del hecho de que las personas quienes participan en intervenciones están interesadas en dejar que los investigadores conozcan su conducción de la vida cotidiana porque las intervenciones instigan sus intereses en reconsiderar y cambiar su conducta de la vida cotidiana.

Este trabajo teórico también ha llevado a nuevas perspectivas sobre la práctica de la psicología. Es mi respuesta a la cuestión: ¿Qué clase de teoría de las personas necesitamos para explicar la clase de cambios del cliente presentado en este escrito? Es una respuesta a los desafíos teóricos que mi estudio y otros parecidos han hecho surgir. Al desarrollar la teoría, he aprendido lecciones importantes al comparar los fenómenos y cuestiones en mi propio proyecto con los fenómenos y asuntos en los otros proyectos en mi grupo de investigación sobre "la conducta personal de la vida cotidiana y la intervención."

Pero la teoría de las personas que yo he delineado apunta a fenómenos de una naturaleza mucho más general -fenómenos que plantean cuestiones respecto a qué significa y que se requiere para ser una persona. Después de todo, los estudios de intervenciones psicológicas no son sino uno de dos principales orígenes de su desarrollo. En términos más básicos, la teoría abre el 
estudio de las dimensiones subjetivas de persona-situación-actividad y ofrece una forma de vincular la investigación sobre la personalidad con la investigación sobre los procesos sociales mediante los cuales las personas conducen sus vidas cotidianas. En este sentido, el escrito argumenta a favor de una aproximación más ecológicamente válida para teorizar sobre la personalidad dentro la psicología de la personalidad.

\section{Referencias}

Baumeister, R. F., Vohs, K. D., \& Funder, D. C. (2007). Psychology as the science of selfreports and finger movements. Perspectives on Psychological Science, 2, 396-403.

Bolger, N., Davis, A., \& Rafaeli, E. (2003). Diary method: Capturing life as it is lived. Annual Review of Psychology, 54, 579-616.

Conner, T. S., Barrett, L., Tugade, M. M., \& Tennen, H. (2007). Idiographic personality. The theory and practice of experience sampling. In R. W. Robins, C. R. Fraley, \& R. F. Krueger, (Eds.), Handbook of research methods in personality psychology (pp. 79-96). New York: Guilford Press.

Costall, A. \& Dreier, O. (Eds.). Doing things with things. The design and use of everyday objects. Aldershot: Ashgate.

Craik, K. (2000). The lived day of an individual: A personenvironment perspective. In W. B. Walsh, K. H. Craik, \& R. H. Price (Eds.), Person-environment psychology: New directions and perspectives (pp. 233-266). Mahwah, NJ: Erlbaum.

Craik, K. H. (2007). Taxonomies, trends, and integrations. In R. W. Robins, C. R. Fraley, \& R. F. Krueger, (Eds.), Handbook of research methods in personality psychology (pp. 209223). New York: Guilford Press.

De Léon, D. (2006). The cognitive biographies of things. In A. Costall \& O. Dreier (Eds.), Doing Things with things. The design and use of everyday objects (pp. 113-130). Aldershot: Ashgate.

Dreier, O. (2008a). Psychotherapy in everyday life. New York: Cambridge University Press.

Dreier, O. (2008b). Learning in structures of social practice. In S. Brinkmann, C. Imholdt, G. Kraft, P. Musaeus, K. Nielsen, \& L. Tanggaard (Eds.), A qualitative stance: Essays in honor of Steinar Kvale (pp. 85-96). Aarhus: Aarhus University Press.

Dreier, O. (2009a). Persons in structures of social practice. Theory \& Psychology, 19, 93-112.

Dreier, O. (2009b). The development of a personal conduct of life in childhood. In T. Teo, P. Stenner, A. Rutherford, E. Park, \& C. Baerveldt (Eds.), Varieties of theoretical psychology: International, philosophical and practical concerns (pp. 175-183). Toronto: Captus Press Inc.

Fleeson, W. (2004). Moving personality beyond the personsituation debate. The challenge and opportunity of withinperson variability. Current Directions in Psychological Science, 13, 83-87.
Fleeson, W. \& Leicht, C. (2006). On delineating and integrating the study of variability and stability in personality psychology: Interpersonal trust as illustration. Journal of Research in Personality, 40, 5-20.

Funder, D. C. (2001). Personality. Annual Review of Psychology, $52,197-221$.

Funder, D. C. (2005). Towards a resolution of the personality triad: Persons, situations, and behaviors. Journal of Research in Personality, 40, 21-34.

Gosling, S. D., Ko, S. J., Mannarelli, T., \& Morris, M. E. (2002). A room with a cue: Personality judgments based on offices and bedrooms. Journal of Personality and Social Psychology, 82, 379-398.

Harré, R. (1998). The singular self. An introduction to the psychology of personhood. London: Sage.

Holzkamp, K. (1995). Alltägliche Lebensführung als subjektwissenschaftliches Grundkonzept. Das Argument 37, 817-846. (Danish translation 1998: Daglig livsførelse som subjektvidenskabeligt grundkoncept. Nordiske Udkast, 26, 3-31.)

Holzkamp, K. (1996). Manuskripte zum Arbeitsprojekt 'Lebensführung'. Forum Kritische Psychologie 36, 7-112. (English translation: Psychology: Social self- understanding on the reasons for action in everyday conduct of life. In U. Osterkamp \& E. Schraube (Eds.) (in press). Turning psychology upside down. Writings of Klaus Holzkamp. Houndmills: Palgrave Macmillan.)

Huniche, L. (2009). Existential concerns in families with Huntington's disease. Towards an understanding of the person in social practice. Theory \& Psychology, 19, 93-113.

Kahneman, D., Krueger, A. B., Schkade, D. A., Schwarz, N., Stone, A. A. (2004). A survey method for characterizing daily life experience: The day reconstruction method. Science, 306, 1776-1780.

Lave, J. (2011). Apprenticeship in critical ethnographic practice. Chicago: University of Chicago Press.

Lewis, M. (2002). Models of development. In D. Cervone \& W. Mischel (Eds.), Advances in personality science (pp. 153-176). New York: The Guilford Press.

Mackrill, T. (2007). Using a cross-contextual qualitative diary design to explore client experiences of psychotherapy. Counselling \& Psychotherapy Research, 7, 233-239.

Mackrill, T. (2008a). Solicited diary studies of psychotherapeutic practice-pros and cons. European Journal of Psychotherapy \& Counselling, 10, 5-18.

Mackrill, T. (2008b). Pre-treatment change in psychotherapy with adult children of problem drinkers: The significance of leaving home. Counselling \& Psychotherapy Research, $8,160-165$.

Mackrill, T. (2008c). Exploring psychotherapy clients' independent strategies for change while in therapy. British Journal of Guidance and Counselling, 36, 441-453.

Mackrill, T. (2011). Differentiating life goals and therapeutic goals: expanding our understanding of the working alliance. British Journal of Guidance \& Counselling, 39, 25-39.

Madsen, K. (2011). Sygdom, pårørende og dagligliv. Når ægtefællen har hjernekræft. [Disease, next-of-kin, 
and everyday life]. Unpublished doctoral dissertation, University of Copenhagen.

McAdams, D. P. (2006). The person. A new introduction to personality psychology (4. ed.). New York: Wiley.

Mehl, M. R., Gosling S. D., \& Pennebaker, J. W. (2006). Personality in its natural habitat: Manifestations and implicit folk theories of personality in daily life. Journal of Personality and Social Psychology, 90, 862-877.

Mischel, W. (1968). Personality and assessment. New York: Wiley.

Mischel, W. (2004). Toward an integrative science of the person. Annual Review of Psychology, 55, 1-22.

Mischel, W. \& Shoda, Y. (2008). Integrating dispositions and processing dynamics within a unified theory of personality. In O. P. John, R. W. Robins, \& L. A. Pervin (Eds.), Handbook of personality. Theory and research. Third Edition (pp. 208-241). New York: The Guilford Press.

Ryle, M. (2010). Screeningsdiagnosticeret sygdom som intervention i den personlige livsførelse. Belyst ved organiseret mammografiscreening i Danmark. [Screening diagnosed disease as intervention in the personal conduct of life. Studied through organized mammography screening in Denmark]. Unpublished doctoral dissertation, University of Copenhagen.
Schatzki, T. R. (2002). The site of the social. A philosophical account of the constitution of social life and change. University Park, PA: Pennsylvania State University Press.

Shoda, Y., Mischel, W., \& Wright, J. C. (1994). Intra-individual stability in the organization of and patterning of behavior: Incorporating psychological situations into the idiographic analysis of personality. Journal of Personality and Social Psychology, 67, 674-687.

Shoda, Y. \& LeeTiernan, S. (2002). What remains invariant?: Finding order within a person's thoughts, feelings, and behaviors across situations. In D. Cervone \& W. Mischel (Eds.), Advances in personality science (pp. 241-270). New York: The Guilford Press.

Smith, D. E. (1987). The everyday world as problematic. Boston: Northeastern University Press.

Sørensen, E. (2009). The materiality of learning. New York: Cambridge University Press.

Wagerman, S. \& Funder, D. C. (2009). Personality psychology of situations. In P. J. Corr \& G. Matthews (Eds.), Cambridge handbook of personality (pp. 27-42). Cambridge: Cambridge University Press.

Weber, M. (1952). The protestant ethic and the spirit of capitalism. New York: Scribner.

Webster's New World College dictionary (1997). Accent Software. Macmillan Publishers. 